\title{
Handedness Shift as a Consequence of Motor Cortex Reorganization After Early Functional Impairment in Left Temporal Lobe Epilepsy-An fMRI Case Report
}

\author{
Pavel Chlebus ${ }^{1,2}$, Milan Brázdil ${ }^{1}$, Petr Hluštík ${ }^{3}$, Michal Mikl ${ }^{1}$, Marta Pažourková2 ${ }^{2}$ Petr Krupa ${ }^{2}$ \\ ${ }_{1}^{1} 1^{\text {st }}$ Department of Neurology, Masaryk University, St. Anne's University Hospital, Brno 656 91, Czech Republic, \\ 2Diagnostic Imaging Clinic, Masaryk University, St. Anne's University Hospital, Brno 656 91, Czech Republic, \\ ${ }^{3}$ Departments of Neurology and Neuroimaging, Olomouc University Hospital, Olomouc 775 20, Czech Republic.
}

\begin{abstract}
The impact of epileptiform activity on the functional organization of various cortical regions is currently a widely discussed topic. Our patient, suffering from left-sided temporal lobe epilepsy with typical mesiotemporal sclerosis, had a remarkable history of a clear-cut handedness shift in early childhood. In this study, we considered the possibility of the functional reorganization of the motor cortex using functional MRI. The results reflect epileptiform activity as an important formative factor for motor cortex development in our patient.
\end{abstract}

\section{Introduction}

Many clinical observations have suggested that the human brain is characterized by functional plasticity. Many studies using various empirical paradigms have also found conclusive evidence for lesion-induced reorganization of speech and motor functions. Epilepsy is not a typical diagnosis mentioned in these studies. The reason may be a lack of clearly defined structural lesions within the cortical regions responsible for these functions. Nevertheless, there is some evidence for a shift of cerebral speech dominance from the left hemisphere to the right one in epileptic patients, including those suffering from left temporal lobe epilepsy (TLE). Wada testing and, more recently, functional imaging studies have been used to determine language lateralization and to study the possible effect of epileptic activity on the plasticity-dependent reorganization of language functions (Adcock et al., 2003; Brázdil et al., 2003; Rey et al., 1998).

Markedly less is known about the reorganization of the motor cortex and the shift of handedness in connection with epileptic disorders. Still, there are some indications of such a process. Former studies report, for example, an atypical proportion of hand preference in the patients with left hemispheric epilepsies and their so-called "pathological left-handedness" (1). Our patient, suffering from left TLE with characteristic mesiotemporal sclerosis, is a typical case; morover, we have direct evidence of handedness shift in the patient's history. We tried to verify this interesting information using fMRI.

\section{Case report}

A 19-year-old female patient was admitted for presurgical assessment of intractable left-sided temporal lobe epilepsy (long-term video-EEG monitoring, high-resolution MRI, interictal PET, Wada procedure). Complex partial seizures had started when the patient was 13 years of age; at the time of our investigation, they occured approximately 1-3 times per month. The patient's birth history and early development were unremarkable, other than febrile seizures at the age of eight months. Subsequent to the febrile seizures, the patient was medicated (phenobarbital) until the age of five, when the medication was suddenly stopped. No clinical seizures were observed during this period. We were credibly informed by the patient's mother that, as a child, the patient unequivocally handled objects with her right hand. At the age of about two years, she spontaneously changed the preference to the left hand and is presently a clear-cut left-hander (according Edinburgh Handedness Inventory). The patient had normal findings in the neurological examination; however, involuntary "mirror" movements of fingers in the non-dominant (right) hand were noted when the patient performed simple voluntary movements with her left hand (Yu et al., 1994). The patient's efforts to suppress the movements minimized them, but she was unable to prevent them completely. A neuropsychological investigation revealed average global intelligence and memory $(\mathrm{IQ}=101, \mathrm{MQ}=107)$. Attention processes were also intact. Long-term video-EEG monitoring (using

Correspondence to: Pavel Chlebus, Stepnicka 1082, Uherske Hradiste 686 06, Czech Republic. Tel: 00420-5-4318-3049; Fax: 00420-5-4318-2624; email: chlepa@centrum.cz 


\section{P. Chlebus et al.}

sphenoidal electrodes) suggested an epileptogenic zone within the left mesiotemporal region. Interictal PET showed decreased blood flow in the left temporal region. High-resolution MRI revealed typical findings of left-sided mesiotemporal sclerosis. The Wada procedure could not be completed due to an unexpected arterial spasm; fMRI was used to determine the hemispheric speech dominance. A silent phonemic verbal fluency task (VFT) was used as a language paradigm (Schlosser et al., 1998). We then evaluated the direction and degree of cerebral lateralization of motor function using fMRI. As a movement paradigm, we chose a sequential finger opposition (SFO) task, in which the patient's thumb sequentially touched each of the four digits at the rate of $1 \mathrm{~Hz}$ (Solodkin et al., 2001). We used an ABAB block design, with active tasks (VFT or SFO) (A) alternating with periods of rest (B). Active as well as control blocks lasted 32 sec. Five blocks of each condition were performed, once for VFT and twice for SFO (with the left and the right hand separately). The patient was studied on a 1.5T scanner (Siemens Symphony). The functional images were acquired using EPI sequences, for both language as well as motor assessment with TR
$($ scan repeat time $)=4520 \mathrm{~ms}, \mathrm{TE}=40 \mathrm{~ms}, \mathrm{FOV}=220 \mathrm{~mm}$, flip angle $90^{\circ}$, matrix size $64 \times 64$, slice thickness $3.5 \mathrm{~mm}$, 32 transversal slices per scan. Each functional measurement consisted of 71 scans. Anatomical T1 weighted images were acquired using a 3D sequence with 160 sagittal slices, resolution $512 \times 512$, slice thickness $1.17 \mathrm{~mm}, \mathrm{TR}=1700 \mathrm{~ms}$, TE $=$ $3.96 \mathrm{~ms}, \mathrm{FOV}=246 \mathrm{~mm}$, flip angle $15^{\circ}$. A statistical parametric mapping (SPM99) program was used to analyze the fMRI data (Friston, 2003). All of the images were preprocessed by default. The final voxel size was $3 \times 3 \times 3 \mathrm{~mm}$, and $\mathrm{p}=0.05$ after correction was set as a threshold for statistical significance of the signal changes in every voxel.

\section{Results}

The pattern of activation in VFT is given in Fig. 1. Atypical (mixed) hemispheric speech dominance with activation maxima (according to volume and statistical significance) in both-sided inferior frontal gyri was proven.

The patterns of activation during the sequential finger opposition task for both hands are given in Fig. 2. The
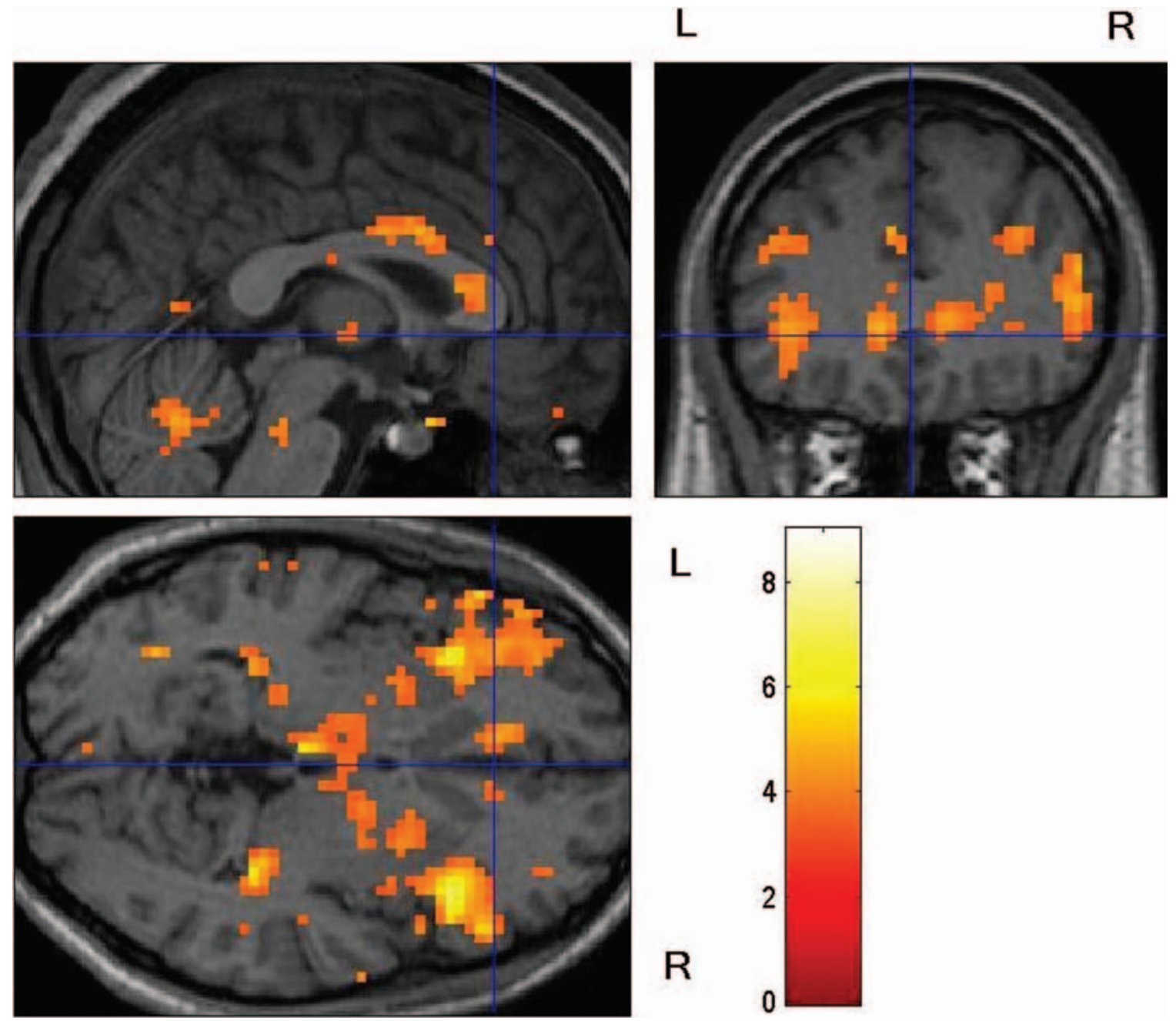

Fig. 1. The pattern of activation during performance of the silent phonemic VFT. Uncorrected $\mathrm{p}=0.001$. 

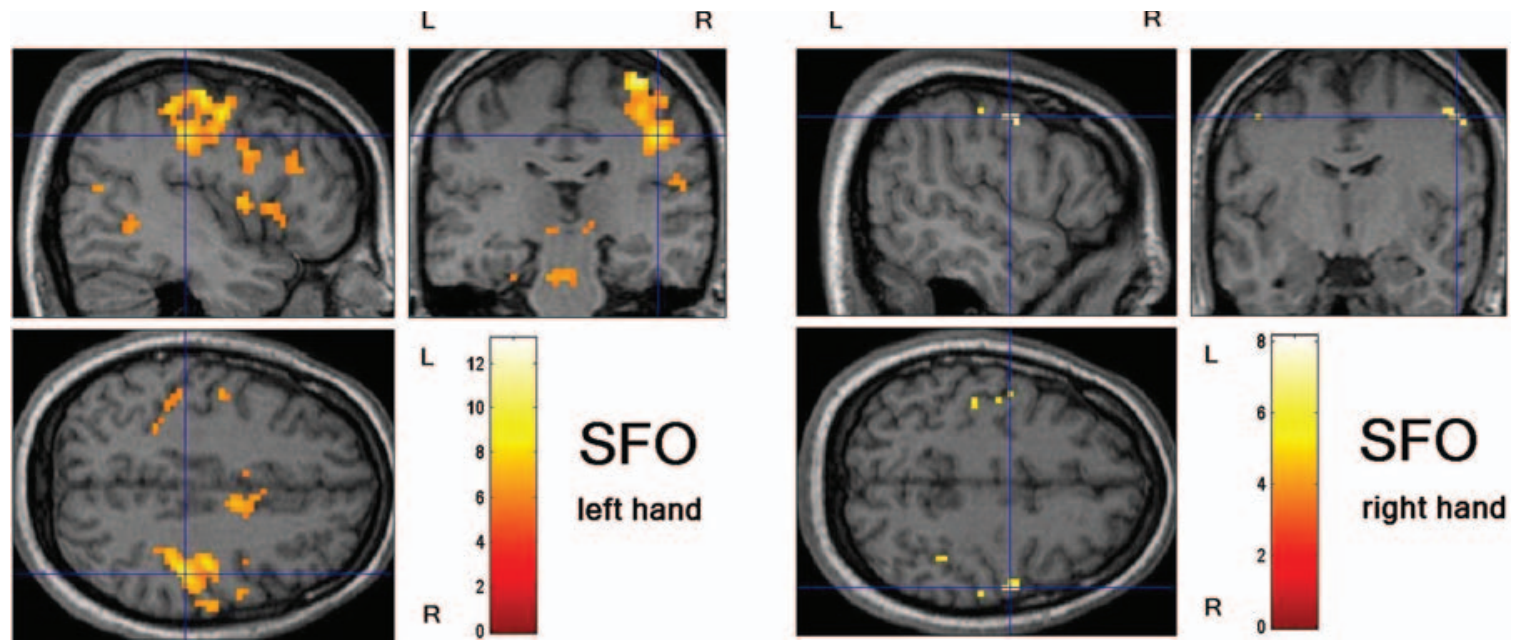

\section{SFO}

left hand

Fig. 2. Patterns of activation during sequential finger opposition of the left (dominant) and right (non-dominant) hand. Corrected $\mathrm{p}=0.05$. Points of intersection in global maxima.

Table 1. The main activated regions during sequential finger opposition of the left (dominant) and right (non-dominant) hand

\begin{tabular}{|c|c|c|c|}
\hline & Region & Volume & Stat. significance \\
\hline \multicolumn{2}{|l|}{ Left (dominant) hand movement } & (in voxels) & (T - statistics) \\
\hline Global maximum & $\begin{array}{l}\text { Right cerebellar cortex - fused with the same region in left } \\
\text { cerebellum activated with lower significance }\end{array}$ & 791 & 13 \\
\hline \multirow[t]{3}{*}{ Local maxima } & $\begin{array}{l}\text { Right primary sensorimotor cortex (BA 2,3,4) fused with a } \\
\text { small activated region of dorsolateral premotor cortex (BA 6) }\end{array}$ & 731 & 12 \\
\hline & Right cingulate gyrus (BA 24, 32) & 118 & 8.9 \\
\hline & Left insular cortex & 80 & 7.8 \\
\hline \multicolumn{4}{|c|}{ Right (non-dominant) hand movement } \\
\hline Global maximum & Right primary sensimotor cortex & 12 & 8.1 \\
\hline \multirow[t]{3}{*}{ Local maxima } & Left cerebellar cortex & 21 & 7.7 \\
\hline & Left primary sensorimotor cortex & 57 & 7.4 \\
\hline & Right cerebellar cortex & 32 & 7.1 \\
\hline
\end{tabular}

expected activation of the contralateral primary sensorimotor cortex (BA 2,3,4) and the ipsilateral cerebellum were dominant during movements of the left (dominant) hand. Only a very subtle activation of the ipsilateral primary sensorimotor area seems to be interesting. A very small activation of the right (contralateral) and no activation of left (ipsilateral) dorsolateral premotor cortex (BA 6) are remarkable. When the patient was moving the right (non-dominant) hand, there was a small but significant activation of the primary sensorimotor cortex bilaterally, with no essential differences in the significance or volume of the activated cortex between the contralateral (left) and ipsilateral (right) hemispheres. The bilateral activation of the cerebellar cortex was also visible. The main activated regions, as well as their volume and statistical significance, are listed in the Table I.

\section{Discussion}

In this study, we used functional MRI to support a hypothesis of the reorganization of the motor cortex and a subsequent shift of handedness in a patient suffering from left-sided TLE. It is not clear whether the behavioural lateralization mani- fested in handedness is directly associated with asymmetry in the activation of cortical motor areas during simple movements. There is strong evidence for a handedness-associated anatomical hemispheric asymmetry. MRI morphometry has shown the central sulcus to be deeper in the dominant than in the non-dominant hemisphere of both left- and right-handed males (Amuts et al., 2000). Microstructural asymmetry of the primary motor cortex has also been observed (Amuts et al., 1997). However, recent functional studies are less conclusive. Asymmetry in the activation of the contralateral primary motor cortex (M1) with the movement-dominant and nondominant hand has been found. But there is not a complete consensus between the results of former studies (Hammond, 2002). A comparison of ipsilateral activations of M1 also fails to present convincing evidence (Li et al., 1996). Other studies have investigated the differences in whole brain activation patterns between movements of the dominant and nondominant hands. These studies typically use motor tasks with higher complexity. Some remarkable differences in activation have been found, mostly involving non-primary cortical regions (i.e. the supplementary motor area, the dorsolateral premotor cortex and the cingulate gyrus) (Hlustik et al., 2002; 
P. Chlebus et al.

Solodkin et al., 2001). During sequential movements, subjects (right- as well as left-handers) showed a variable degree of activation in both ipsilateral and contralateral areas (involving M1 and motor association areas) using the dominant as well as the non-dominant hand. Our patient was not an exception, but the pattern of activation was quite different from dextrals and non-pathological sinistrals as well. The most surprising finding seems to be the generally small activation of the dorsolateral premotor cortex (the function of this area is related to complexity of movement, and the significance of activation is often used to estimate the direction and the degree of handedness) $(6,12)$. Bilateral activation of cerebellar cortex also seems unusual and could testify for functional recovery (Chollet et al., 1991). Recruitment of the anterior insula can be related to motor cortex reorganization as well (Small et al., 2002).

This study, in the context of other recently published papers, does not permit a define decision about handedness or even about handedness shift. However, several atypical findings present evidence of changes in the cortical motor representation: First, we observed atypical hemispheric speech dominance according to functional MRI results. Second, we observed involuntary mirror movements of fingers in the non-dominant (right) hand while the patient performed SFO with the left hand. These mirror movements can be seen frequently in hemiparetic patients with early left hemispheric damage (4). Considering the clinical history together with the atypical pattern of motor cortex activation, we can view epileptic activity as an important forming factor for motor cortex development in our patient. We can only speculate about the possible role of "the disruptive effect of spreading epileptiform activity on the cortical association areas" (Rausch and Walsh, 1984). This spread does not affect the primary motor cortex, but it could have influence on the formation of the motor circuits responsible for motor skills. Thus, if the primary dominant hand (right) was unable to develop into the dominant extremity, the left hand became more "dexterous" and assumed the dominant role. Understanding the principles of brain plasticity remains seriously limited, but fMRI methods give us "a hand" in the pursuit of this fascinating goal.

\section{References}

Adcock JE, Wise RG, Oxbury JM, Oxbury SM, Mathews PM. Quantitative fMRI assessment of the differences in lateralization of language-related brain activation in patients with temporal lobe epilepsy. Neuroimage 2003; 18: 423-38.

Amunts K, Jancke L, Mohlberg H, Steinmetz H, Zilles K. Interhemispheric asymmetry of the human motor cortex related to handedness and gender. Neuropsychologia 2000; 38: 304-12.

Amunts K, Schmidt-Passos F, Schleicher A, Zilles K. Postnatal development of interhemispheric asymmetry in the cytoarchitecture of human area 4. Anat Embryol 1977; 196: 393-402.

Brázdil M, Zákopčan J, Kuba R, Fanfrdlová Z, Rektor I. Atypical hemispheric language dominance in left temporal lobe epilepsy as a result of the reorganization of language functions. Epilepsy Behav 2003; in press

Chollet F, DiPiero V, Wise RJS, Brooks DJ, Dolan RJ, Frackowiak RSJ .The functional anatomy of motor recovery after stroke in humans: A study with positron emission tomography. Ann Neurol 1991; 29: 63-71.

Friston KJ. SPM99. Welcome Department of Cognitive Neurology, Institute of Neurology, University College London. Programme available on http: llwww. fin.ion.ucl.ac.uklspm\ (June 2003).

Hammond G. Correlates of human handedness in primary motor cortex: A review and hypothesis. Neurosci Biobehav Rev 2002; 26: 285-92.

Hlustik P, Solodkin A, Gullapalli RP, Noll DC, Small SL. Functional lateralization of the human premotor cortex during sequential movements. Brain Cogn 2002; 49: 54-62.

Li A, Yetkin FZ, Cox R, Haughton VM. Ipsilateral hemisphere activation during motor and sensory tasks. Am J Neuroradiol 1996; 17: 651-55.

Rausch R, Walsh GO. Right-hemisphere language dominance in right handed epileptic patients. Arch Neurol 1984; 41: 1077-80.

Rey M, Dellatolas G, Bancaud J, Talairach J. Hemispheric lateralization of motor and speech functions after early brain lesion: Study of 73 epileptic patients with intracarotid amytal test. Neuropsychologia 1988; 26: 167-72.

Schlosser R, Hutchinson M, Jossefer S. Functional magnetic resonance imaging of human brain activity in a verbal fluency task. J Neurol Neurosurg Psychiatry 1998; 64: 492-98.

Small SL, Hlustik P, Noll DC, Genovese C, Solodkin A. Cerebellar hemispheric activation ipsilateral to the paretic hand correlates with functional recovery after stroke. Brain 2002; 125: 1544-57.

Solodkin A, Hlustik P, Noll DC, Small SL. Lateralization of motor circuits and handedness during finger movements. Eur J Neurol 2001; 8: 425-34.

Yue C, Vikingstad EM, Huttenlocher PR, Towle VL. Functional magnetic resonance studies of the reorganization of the human hand sensorimotor area after unilateral brain injury in the perinatal period. Proc Natl Acad Sci 1994; 91: 9612-16. 